\title{
Ovarian biomarkers predict controlled ovarian stimulation for in vitro fertilisation treatment in Singapore
}

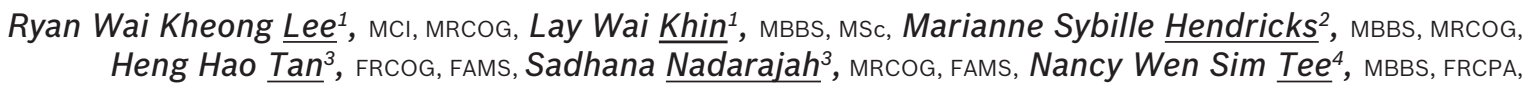
Seong-Feei $\underline{\mathrm{Loh}}^{2}$, FRCOG, FAMS, Bee Choo $\underline{\mathrm{Ta}}^{5,6}$, PhD, Jerry KY $\underline{\mathrm{Chan}}^{3,7}$, PhD, FRCOG

INTRODUCTION Ovarian biomarkers have been shown to predict responses to controlled ovarian hyperstimulation (COH) during in vitro fertilisation (IVF) in predominantly Caucasian populations, with limited studies performed in Southeast Asian women in Singapore.

METHODS We evaluated the performance of serum anti-Müllerian hormone (AMH), follicle-stimulating hormone and oestradiol levels, antral follicle count (AFC), body mass index, ovarian volume, and age to establish thresholds for the prediction of poor (< 4 oocytes retrieved) and excessive responses ( $>19$ oocytes retrieved) in 263 women undergoing $\mathrm{COH}$. Univariate and multivariate logistic regression analysis and receiver operating characteristic curves were used to calculate probabilities for poor and excessive responders to $\mathrm{COH}$.

RESULTS $36(13.7 \%)$ and 50 (19.0\%) women had poor and excessive response to COH, respectively. An AMH value of $0.69 \mathrm{ng} / \mathrm{mL}$ predicted poor ovarian response with positive likelihood ratio (LR) of 2.94, compared to an AFC of $\leq 5$ when the positive $L R$ is 2.36 . Conversely, an $A M H$ value of $\geq 3.06 \mathrm{ng} / \mathrm{mL}$ predicted excessive ovarian response with positive $L R$ of 2.24, compared to an AFC cut-off of $\geq 12$ with positive LR of 1.93 .

CONCLUSION AMH levels and AFC are equivalent in the prediction of both poor and excessive ovarian response in women undergoing IVF. Our study highlights the importance of establishing population-specific cut-off biomarker values so that protocols can be tailored to optimise IVF treatment.

Keywords: biomarkers, fertilisation, ovarian, stimulation

\section{INTRODUCTION}

Ovarian reserve markers serve as an important tool in planning in vitro fertilisation (IVF) stimulation cycles in order to achieve an optimal number of oocytes for fertilisation. For example, a predicted high responder may be started on an antagonist cycle with a low follicle-stimulating hormone (FSH) dose and possibly an antagonist trigger for final oocyte maturation to reduce, albeit not eliminate, the chances of ovarian hyperstimulation syndrome (OHSS). ${ }^{(1,2)}$ Conversely, for predicted poor responders or patients with diminished ovarian reserves, appropriate counselling on the possibility of cycle cancellation and an appropriate starting dose of FSH with adjuvants such as growth hormone may be considered. ${ }^{(3)}$

Common ovarian reserve markers used include age, early FSH and oestradiol (E2) levels, body mass index (BMI), ovarian volume, and antral follicle count (AFC). ${ }^{(3)}$ Of these, AFC has the highest sensitivity in determining ovarian response. ${ }^{(4)}$ More recently, a slew of studies have shown that serum anti-Müllerian hormone (AMH) has similar performance as AFC in identifying both poor and excessive responders in patients undergoing controlled ovarian hyperstimulation $(\mathrm{COH})$ during IVF or intracytoplasmic sperm injection (ICSI) treatment. ${ }^{(5-7)}$ Both AMH level and AFC also demonstrate a linear relationship with oocyte yields and have a strong correlation with each other. ${ }^{(8,9)}$
There are limited studies on ovarian reserve markers in response to controlled ovarian stimulation in multi-ethnic Southeast Asian women in Singapore. ${ }^{(10,11)}$ This may be important, as it has been shown that $\mathrm{AMH}$ level may be influenced by ethnicity. For example, AMH levels can be 25\% lower in AfroAmerican and Hispanic women compared to Caucasians, with genetic polymorphism being implicated. ${ }^{(12,13)}$ In addition, $\mathrm{FSH}$ receptor polymorphisms affect different ethnicities, which may play a role in the prediction of ovarian response. ${ }^{(14)} \mathrm{AMH}$ measurements can also be affected by differences between generations of immunoassays; both technical aspects and a lack of standardisation between these assays may affect reliability and interpretation of AMH level results. ${ }^{(15-17)}$

Accordingly, we evaluated the performance of serum $\mathrm{AMH}$, early follicular phase Day 2 or 3 FSH and E2 levels, AFC, BMI, ovarian volume and age as biomarkers for ovarian response to IVF treatment, and established thresholds for the prediction of poor and excessive responses to $\mathrm{COH}$ in patients undergoing IVF.

\section{METHODS}

Women undergoing fresh IVF/ICSI cycles at KK Women's and Children's Hospital in Singapore were prospectively recruited from March 2009 to January 2012. This study was approved by the SingHealth Centralised Institutional Review Board,

\footnotetext{
${ }^{1}$ Department of Obstetrics and Gynaecology, KK Women's and Children's Hospital, ${ }^{2}$ The O\&G Specialist Clinic, ${ }^{3}$ Department of Reproductive Medicine, ${ }^{4}$ Department of Pathology and Laboratory Medicine, KK Women's and Children's Hospital, ${ }^{5}$ Saw Swee Hock School of Public Health, ${ }^{6}$ NUS Yong Loo Lin School of Medicine, National University of Singapore, 'Programme in Cancer and Stem Cell Biology, Duke-NUS Medical School, Singapore

Correspondence: Dr Ryan Lee Wai Kheong, Associate Consultant, Department of Obstetrics and Gynaecology, KK Women's and Children's Hospital, 100 Bukit Timah Road, Singapore 229899. Ryan.lee.wai.kheong@singhealth.com.sg
} 
Singapore. The patients' past medical history and reproductive treatments were noted. All patients underwent a general physical and gynaecological examination, as well as ultrasonography of the pelvis. Human semen analysis was performed for the patients' male partners prior to IVF/ICSI. We included women with primary or secondary subfertility diagnoses of: male factor, tubal factors, anovulatory cycles, endometriosis or unexplained fertility. Women who were more than 45 years of age or those with endocrine disorders such as diabetes mellitus and thyroid dysfunction were excluded.

After obtaining informed consent, all women underwent serum blood sampling to measure serum levels of $\mathrm{AMH}$ and $\mathrm{FSH}$ on Day 2 or 3 of the menstrual cycle. All samples were spun down immediately, stored in aliquots at $-20^{\circ} \mathrm{C}$ and batch tested twice weekly on site at KK Women's and Children's Hospital using an in-house enzyme-linked immunosorbent assay (ELISA) method (AMH Beckman DSL kit, Webster, TX, USA), with inter- and intra-variability of $5.4 \%$ and $5.6 \%$, respectively. Transvaginal two-dimensional ultrasonography was used (Aloka, Prosound $\alpha 7$; Hitachi Aloka Medical America, CT, USA) to determine AFC and ovarian volume prior to commencement of the IVF cycle. AFC was defined as the total number of follicles in both ovaries before the start of stimulation with an average diameter of two orthogonal planes measuring 2-9 $\mathrm{mm}$ on transvaginal ultrasonography, as described previously. ${ }^{(18)}$ Serum AMH and AFC results were not used to determinine the starting dose of recombinant FSH used in the IVF/ICSI cycle.

All women underwent standard $\mathrm{COH}$ protocols employing either long agonist or short antagonist cycles with or without the use of combined oral contraceptive (COC) pills in a crossover protocol in anovulatory women, as determined by their own doctors. Women undergoing COC crossover commenced Microgynon 30 (ethinyloestradiol $30 \mathrm{mcg}$, levonorgestrel $150 \mathrm{mcg}$ ) on Days 1-2 of menses of the previous cycle for 14-16 days with a washout period of five days before crossing over to the agonist or antagonist cycle, as determined by their own doctors. Women undergoing the long gonadotropin-releasing hormone $(\mathrm{GnRH})$ agonist protocol were treated with $\mathrm{GnRH}$ agonist $(0.5 \mathrm{mg}$ leuprorelin acetate; AbbVie, North Chicago, IL, USA) for 14 days, starting from menstrual cycle Day 21 or after completion of 21 days of COC. Serum E2 level $<50 \mathrm{pmol} / \mathrm{mL}$ on cycle Day 2 and endometrial thickness $<4 \mathrm{~mm}$ indicated adequate pituitary supression before $\mathrm{COH}$ was initiated with recombinant FSH (follitropin beta, Puregon; Schering-Plough, Kenilworth, NJ, USA) at different age-specific doses ( $<35$ years: $200 \mathrm{U}$ per day, 35-38 years: $250 \cup$ per day and $>38$ years: $300 \cup$ daily), with a lower dose of $150 \mathrm{U}$ daily for women diagnosed with polycystic ovarian syndrome. Women undergoing the $\mathrm{GnRH}$ antagonist protocol commenced $\mathrm{COH}$ with recombinant $\mathrm{FSH}$ on menstrual cycle Days 2-4, with the addition of daily $\mathrm{GnRH}$ antagonist (0.25 mg ganirelix acetate; Merck Sharp \& Dohme, Kenilworth, NJ, USA) from Day 5 or 6 of stimulation until the day of human chorionic gonadotropin (hCG) trigger. hCG trigger (intramuscular 10,000 U hCG, pregnyl; Merck Sharp \& Dohme) was administered for final oocyte maturation when $\geq 3$ follicles of $\geq 17 \mathrm{~mm}$ were observed. Transvaginal oocyte retrieval was performed under sedation 34-46 hours after hCG trigger. Retrieved metaphase II stage mature oocytes were fertillised by either IVF or ICSI, and embryo transfer was performed 48-72 hours after oocyte retrieval. Two embryos were transferred and serum beta-hCG was measured on Day 17 after embryo transfer to confirm the pregnancy. Women received Utrogestan (micronised progesterone; Besins Healthcare, Paris, France) $200 \mathrm{mg}$ thrice daily for luteal phase support until serum beta-hCG was measured. Clinical pregnancy was defined as the presence of positive fetal cardiac activity and intrauterine gestation sac detection on ultrasonography at six weeks of pregnancy.

The primary objective of the study was to predict poor and excessive ovarian response to $\mathrm{COH}$ from individual biomarkers using serum AMH level, AFC, early FSH level, E2 level, BMI, ovarian volume and age. The secondary objective was to establish threshold values of ovarian markers in poor and excessive ovarian response to $\mathrm{COH}$. The ovarian response was defined according to the number of oocytes retrieved. Poor ovarian response was defined as cancellation of cycles with $>2$ follicles of $>11 \mathrm{~mm}$ in diameter, or $<4$ oocytes retrieved at oocyte retrieval. ${ }^{(19)}$ Excessive ovarian response was defined as $>19$ oocytes retrieved during oocyte retrieval. ${ }^{(20)}$

Categorical variables were presented as numbers and percentages with $95 \%$ confidence interval $(\mathrm{Cl})$. Continuous variables were presented as mean \pm standard deviation or median and range, depending on the distribution of data. To evaluate the significant independent predictors for poor and excessive responders, univariate and multivariate logistic regression with stepwise backward procedure was performed. The final model selection was carried out based on the likelihood ratio (LR) test. We also assessed the discrimination ability among logistic regression prediction models using net reclassification improvement (NRI) as well as indices of improvement in discrimination (IDI) statistics. The significance level was $\alpha=0.05$ in a two-sided test.

To compare the predictive abilities of different biomarkers for poor and excessive responders, receiver operating characteristic (ROC) curves were constructed to identify the global accuracy (area under the curve $[\mathrm{AUC}]$ ) of our study covariates of interest (age, AFC, AMH and FSH level) based on predicted probability values, which was calculated using a post-estimation command after multivariate logistic regression with STATA statistical software Release 11 (StataCorp, College Station, TX, USA). The AUCs were estimated by points and $95 \% \mathrm{Cl}$ and compared using C-statistics.

Ideal cut-off points for age, AMH level, AFC and FSH level were determined using ROC curve and other statistics for any classification method. The 'rocss' command in STATA was used to calculate sensitivity and specificity using calculated probabilities from logistic regression analyses. Positive LR (LR+) and negative LR (LR-) were also calculated for all biomarkers to predict pre-specified clinical responses (poor and excessive responders). A two-sided $p$-value $<0.05$ indicated statistical significance. A correlation matrix on all seven biomarkers was obtained with STATA. 


\section{RESULTS}

A total of 263 women with a mean age of $35.0 \pm 3.8$ years were recruited for this study. The ethnicity of the study population was reflective of the demographics in Singapore, with the majority (76.4\%) of the participants being Chinese. The main characteristics of this population, including their infertility diagnoses, are shown in Table I. $76.4 \%$ of our cohort underwent the long agonist cycle, with $\mathrm{FSH}$ dosage based upon age. None of the cycles were cancelled prior to oocyte retrieval in this cohort. There were $81(31.4 \%)$ clinical pregnancies in the stimulated cycle among 258 women for whom data was available; five women had an unknown pregnancy status as they defaulted from pregnancy blood tests and subsequent ultrasonography.

$36(13.7 \%)$ out of 263 women in the cohort had a poor response to $\mathrm{COH}$ with $<4$ oocytes retrieved at oocyte retrieval, and $50(19.0 \%)$ had an excessive response of $\geq 20$ oocytes retrieved after $\mathrm{COH}$. The AMH quartile range was $<0.80$, $0.80-4.75$ and $>4.75$ for the $<25$ percentile, $25-75$ percentile and $>75$ percentile ranges, respectively.

Univariate logistic regression analysis showed that only AMH level, AFC, Day 2/3 FSH level and age were independent significant predictors of poor response (Table II). On multivariate stepwise logistic regression analysis, the final fitted model using age, AMH level and AFC did not show any significant improvement (AUC 0.88, p > 0.05 based on IDI and NRI statistics) in discriminant ability and NRI. By plotting the AUC for the prediction of poor response according to the European Society of Human Reproduction and Embryology consensus of fewer than four oocytes retrieved, ${ }^{(19)}$ the best performers were $\mathrm{AMH}$

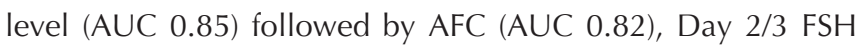

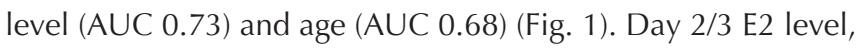
ovarian volume (two- and three-dimensional) and BMI were poor predictors of poor response with AUC of 0.65, 0.61 and 0.53, respectively. The sensitivities, specificities and LRs for positive and negative test values of each biomarker for predicting a poor response are detailed in Table II. An AMH cut-off value of $\leq 0.69$ $\mathrm{ng} / \mathrm{mL}$ predicted poor ovarian response with $70.6 \%$ sensitivity, $76.0 \%$ specificity and a positive LR of 2.94, compared to an AFC cut-off value of $\leq 5$ with $75.0 \%$ sensitivity, $68.2 \%$ specificity and positive LR of 2.36.

AMH level, AFC, Day 2/3 FSH level and age were independent significant predictors for excessive ovarian response on univariate logistic regression analysis (Table III). The best predictors for excessive response were AFC (AUC 0.81),

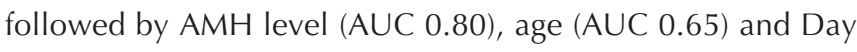
2/3 FSH level (AUC 0.63) (Fig. 2). On multivariate stepwise logistic regression analysis, the final fitted model using age, $\mathrm{AMH}$ level and AFC did not improve on the prediction (AUC $0.83, p>0.05$ based on IDI and NRI statistics). An AMH cutoff value of $>3.06 \mathrm{ng} / \mathrm{mL}$ predicted excessive ovarian response with $76.0 \%$ sensitivity, $66.2 \%$ specificity and a positive LR of 2.24 , while an AFC cut-off of $\geq 12$ had $72.0 \%$ sensitivity, $63.0 \%$ specificity with a positive LR of 1.93 for predicting excessive ovarian response (Table III).
Table I. Demographic and clinical characteristics of the patients with oocytes retrieved during in vitro fertilisation $(n=263)$.

\begin{tabular}{|c|c|}
\hline Parameter & No. (\%)/mean \pm SD \\
\hline Age (yr) & $35.0 \pm 3.8$ \\
\hline$<31$ & $21(8.0)$ \\
\hline $31-37$ & $168(63.9)$ \\
\hline$>37$ & $74(28.1)$ \\
\hline \multicolumn{2}{|l|}{ Ethnicity } \\
\hline Chinese & $201(76.4)$ \\
\hline Malay & $19(7.2)$ \\
\hline Indian & $25(9.5)$ \\
\hline Other & $18(6.8)$ \\
\hline BMI $\left(\mathbf{k g} / \mathbf{m}^{2}\right)$ & $22.2 \pm 4.1$ \\
\hline$<26$ & $215(81.7)$ \\
\hline $26-30$ & $34(12.9)$ \\
\hline$>30$ & $14(5.3)$ \\
\hline Mean AMH (ng/mL) & $3.36 \pm 2.82$ \\
\hline Median AMH* (ng/mL) & $2.67(0.19-14.28)$ \\
\hline \multicolumn{2}{|c|}{ AMH quartile range (ng/mL) } \\
\hline$<0.80$ & $61(23.2)$ \\
\hline $0.80-4.75$ & $136(51.7)$ \\
\hline$>4.75$ & $66(25.1)$ \\
\hline \multicolumn{2}{|l|}{ Causes of subfertility } \\
\hline Male factor & $26(9.9)$ \\
\hline Tubal factor & $48(18.3)$ \\
\hline Ovarian disorder & $38(14.4)$ \\
\hline Unexplained & $86(32.7)$ \\
\hline Endometriosis & $65(24.7)$ \\
\hline \multicolumn{2}{|l|}{ Type of stimulation cycle } \\
\hline Long GnRH agonist & $201(76.4)$ \\
\hline Short GnRH antagonist & $62(23.6)$ \\
\hline \multicolumn{2}{|l|}{ No. of oocytes recovered } \\
\hline$\geq 4$ & $227(86.3)$ \\
\hline$<4$ & $36(13.7)$ \\
\hline$\leq 19$ & $213(81.0)$ \\
\hline$>19$ & $50(19.0)$ \\
\hline
\end{tabular}

*Data presented as median (range). AMH: anti-Müllerian hormone; BMI: body mass index; GnRH: gonadotropin-releasing hormone; SD: standard deviation

\section{DISCUSSION}

This prospective cohort study is one of a limited number of studies examining predictors of ovarian response in women undergoing IVF in a multi-ethnic Southeast Asian population in Singapore. Out of the seven possible markers, we found that $\mathrm{AMH}$ level and AFC are broadly equivalent in predicting both poor and excessive ovarian response in women undergoing IVF, in congruence with other studies published elsewhere.

ROC analyses showed that AMH level and AFC are the two better predictors of poor ovarian response compared to age, FSH level, E2 level, BMI and ovarian volume. Our finding that an AMH cut-off of $\leq 0.69 \mathrm{ng} / \mathrm{mL}$ and an AFC $\leq 5$ were the best predictors for poor response is in broad agreement with a recent meta-analysis done by the IMPORT study group demonstrating the utility of using AMH level and AFC to predict poor ovarian 
Table II. Univariate logistic regression analysis and sensitivity, specificity and likelihood ratios for the prediction of poor response at different clinical cut-off values.

\begin{tabular}{|llllllll|}
\hline Parameter & Cut-off & AUC & Sensitivity & Specificity & LR+ & LR- & p-value \\
\hline AMH $(\mathrm{ng} / \mathrm{mL})$ & $\leq 0.69$ & 0.85 & 70.6 & 76.0 & 2.94 & 0.39 & $<0.001$ \\
\hline AFC & $\leq 5$ & 0.82 & 75.0 & 68.2 & 2.36 & 0.37 & 0.001 \\
\hline Day 2/3 FSH (IU/L) & $\geq 5.7$ & 0.73 & 72.0 & 80.5 & 1.30 & 0.64 & 0.001 \\
\hline Age $(\mathrm{yr})$ & $\geq 35$ & 0.68 & 69.6 & 80.3 & 1.30 & 0.70 & 0.002 \\
\hline E2 $(\mathrm{pmol} / \mathrm{mL})$ & $\geq 82$ & 0.66 & 70.5 & 24.1 & 0.93 & 1.22 & 0.158 \\
\hline Ovarian volume $\left(\mathrm{cm}^{3}\right)$ & $\geq 10.6$ & 0.61 & 70.4 & 22.3 & 0.90 & 1.33 & 0.095 \\
\hline BMI $\left(\mathrm{kg} / \mathrm{m}^{2}\right)$ & $\geq 25$ & 0.54 & 17.1 & 82.1 & 0.98 & 1.00 & 0.216 \\
\hline
\end{tabular}

AFC: antral follicle count; AMH: anti-Müllerian hormone; AUC: area under the curve; BMI: body mass index; E2: oestradiol; FSH: follicle-stimulating hormone; LR+: positive likelihood ratio; LR-: negative likelihood ratio

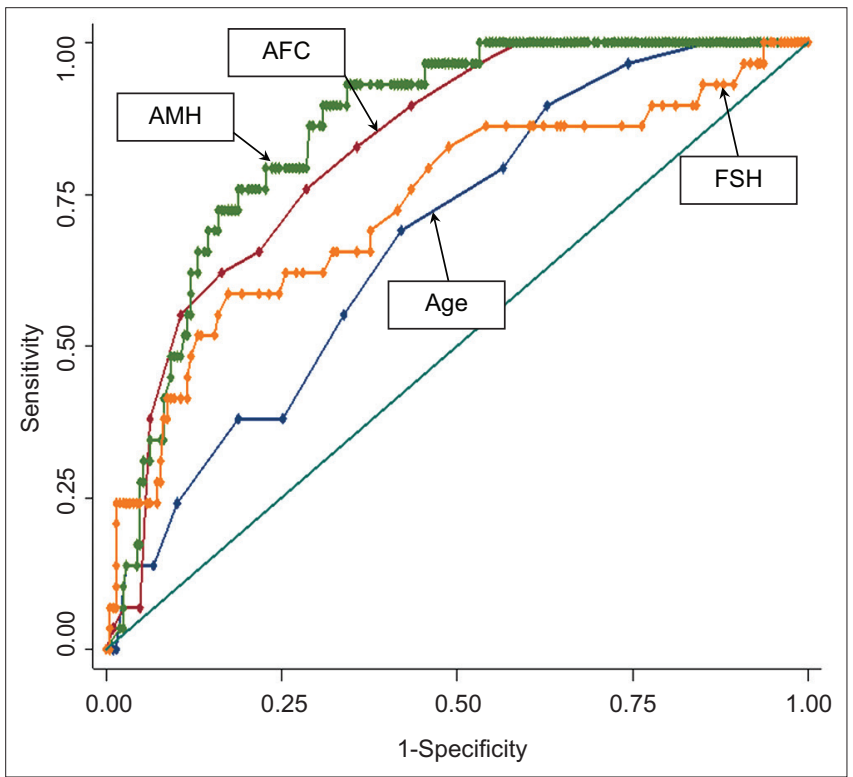

Fig. 1 Receiver operating characteristic curve shows the predictive ability of antral follicle count (AFC), anti-Müllerian hormone (AMH), age and folliclestimulating hormone (FSH) for poor responders (based on probability calculated from univariate logistic regression).

response in women undergoing IVF treatment. ${ }^{(20)}$ Our cut-off of $\leq 0.69 \mathrm{ng} / \mathrm{mL}(5 \mathrm{pmol} / \mathrm{L})$ for the prediction of poor response is similar to those established by other groups. ${ }^{(21,22)}$

Reichman et al have also shown that an $\mathrm{AMH}$ level of $<0.7 \mathrm{ng} / \mathrm{mL}$ was associated with significantly higher cycle cancellation rates compared to an AMH level of $>2.01 \mathrm{ng} / \mathrm{mL}$. ${ }^{(22)}$ In contrast, a recent homogeneous Vietnamese cohort study by Lan et al suggested a higher cut-off of $<1.38 \mathrm{ng} / \mathrm{mL}$ to predict poor response (AUC 0.88). However, it is not known how AMH levels were measured in that study. ${ }^{(23)}$ In our study, the corresponding value for prediction of poor response using AFC was $\leq 5$, which

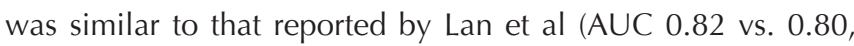
respectively). ${ }^{(23)}$ Thus, the high $\mathrm{LRs}$ associated with both $\mathrm{AMH}$ level and AFC suggest their clinical utility in predicting a poor response to $\mathrm{COH}$.

To assess excessive response to $\mathrm{COH}$, we selected an ovarian response of $>19$ oocytes retrieved as a criteria for hyper-response, as this has been associated with a clinically important sixfold increase in moderate-to-severe OHSS at our centre for women who required admission $(16.6 \%$ vs. $2.6 \%$, unpublished data from 2008). Several other studies typically used the cut-off of
$>15$ oocytes. ${ }^{(20,24-26)}$ Our findings closely mirror those from the IMPORT study group, where AMH level (AUC 0.80 vs. 0.81),

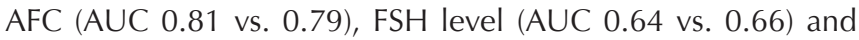
age (AUC 0.65 vs. 0.61 ) were found to be useful predictors for excessive response (> 15 oocytes in the IMPORT study group). ${ }^{(27)}$ In another study, Lee et al showed that an AMH cut-off level of $3.36 \mathrm{ng} / \mathrm{mL}$ is highly predictive for the development of OHSS in a Chinese population in Taiwan. ${ }^{(28)}$ This level was similar to our cut-off of $3.06 \mathrm{ng} / \mathrm{mL}$, although we did not use OHSS as an endpoint. Lan et al showed that both AMH level and AFC are good predictors for excessive response to $\mathrm{COH}$ at a lower cutoff value of $2.1 \mathrm{ng} / \mathrm{mL}$ for AMH (AUC 0.76) and a similar AFC cut-off of 12.25 (AUC 0.81). ${ }^{(23)}$ Our data is also consistent with a recent local study by Lee et al showing that $\mathrm{AMH}$ level and AFC were reliable markers for prediction of ovarian response to COS, although the cut-off levels differ. ${ }^{(10)}$ This may be due to the use of different definitions of poor ovarian response $(\leq 4$ oocytes vs. $<4$ oocytes) and AFC measurements (2-10 mm vs. 2-9 mm) in their study compared to the present study.

The interpretation of $\mathrm{AMH}$ results may be affected by the various assays used. The first-generation AMH ELISA assay 
Table III. Predictors of hyper-responders using sensitivity, specificity and likelihood ratios according to clinical cut-off values, and the p-value of univariate logistic regression analysis.

\begin{tabular}{|llllllll|}
\hline Parameter & Value & AUC & Sensitivity & Specificity & LR+ & LR- & p-value \\
\hline AFC & $\geq 12$ & 0.81 & 72.0 & 63.0 & 1.93 & 0.44 & 0.001 \\
\hline AMH $(\mathrm{ng} / \mathrm{mL})$ & $\geq 3.06$ & 0.80 & 76.0 & 66.2 & 2.24 & 0.35 & 0.001 \\
\hline Age $(\mathrm{yr})$ & $\leq 35$ & 0.65 & 70.1 & 76.9 & 0.58 & 0.38 & 0.002 \\
\hline Day $2 / 3 \mathrm{FSH}$ & $\leq 4.5$ & 0.63 & 80.0 & 94.1 & 0.88 & 0.21 & 0.001 \\
\hline Ovarian volume $\left(\mathrm{cm}^{3}\right)$ & $\geq 13.6$ & 0.61 & 72.0 & 52.8 & 1.53 & 0.53 & 0.232 \\
\hline BMI $\left(\mathrm{kg} / \mathrm{m}^{2}\right)$ & $\geq 30$ & 0.52 & 6.0 & 94.5 & 1.07 & 0.99 & 0.351 \\
\hline E2 $(\mathrm{pmol} / \mathrm{mL})$ & $\leq 87$ & 0.52 & 71.0 & 33.0 & 1.06 & 0.88 & 0.353 \\
\hline
\end{tabular}

AFC: antral follicle count; AMH: anti-Müllerian hormone; AUC: area under the curve; BMI: body mass index; E2: oestradiol; FSH: follicle-stimulating hormone; LR+: positive likelihood ratio; LR-: negative likelihood ratio

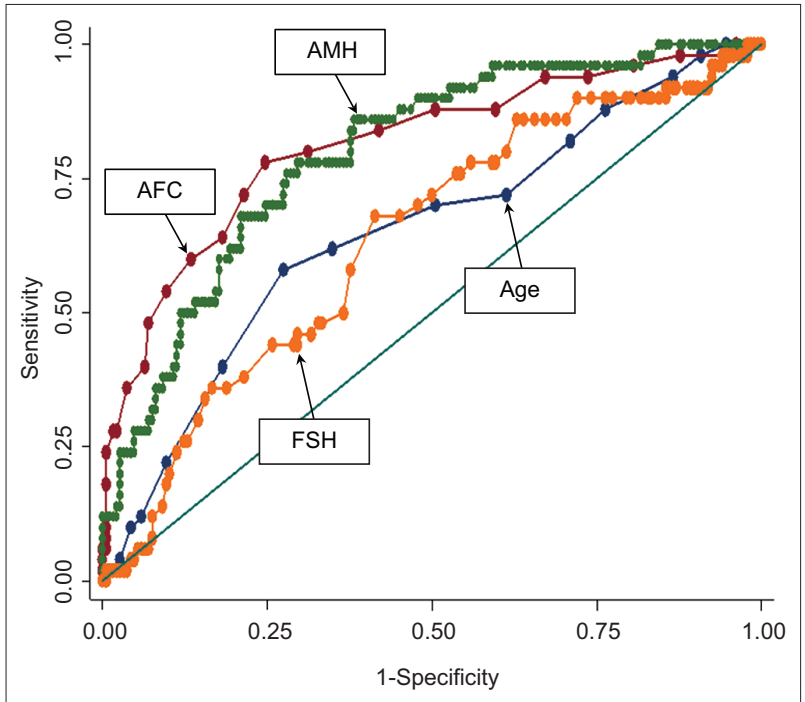

Fig. 2 Receiver operating characteristic curve shows the predictive ability of antral follicle count (AFC), anti-Müllerian hormone (AMH), age and folliclestimulating hormone (FSH) for excessive responders (based on probability calculated from univariate logistic regression).

by Diagnostic Systems Laboratories (Webster, TX, USA) and Immunotech assay were replaced by the second-generation $\mathrm{AMH}$ Beckman Coulter, since 2011. ${ }^{(15,16,29)}$ Our study was performed using the first-generation Diagnostic Systems Laboratories assay rather than the second-generation assays used in other studies, which may affect the comparison and interpretation of $\mathrm{AMH}$ readings. Recent publications have reported a discrepancy in $\mathrm{AMH}$ values obtained using the second-generation assay compared to those obtained with the Immunotech and Diagnostic Systems Laboratories assays. ${ }^{(29,30)}$ The use of different assays should be taken into consideration when interpreting these measurements.

In our study, AMH levels and AFC results were not used to determine the dosage of FSH that patients received. Patients underwent IVF stimulation cycles based on their doctor's clinical decision. The incorporation of $\mathrm{AMH}$ and/or AFC in tailoring IVF stimulation cycles may indeed allow an optimal ovarian response to be attained. ${ }^{(31,32)}$ This allows us to tailor IVF stimulation regimens based on a patient's AMH level, AFC and age to optimise an adequate response to COS and to prognosticate patients appropriately. As AFC and AMH levels decline over age, age-related nomograms can be used as a reference to tailor the ovarian stimulation regime in IVF. ${ }^{(11)}$
The ovarian reserve markers in our study had broadly similar measurements across the different ethnic groups in our study. However, we acknowledge that the small number $(n=62)$ of patients from minority ethnic groups limited our ability to draw conclusions about interethnic group differences.

In conclusion, AMH level and AFC are good predictors of ovarian response in Southeast Asian women undergoing IVF in Singapore. We did not find BMI, ovarian volume and early follicular phase E2 level to be useful predictors of ovarian response to $\mathrm{COH}$. The observed differences in poor and excessive responders with other published studies suggest that centre- and population-specific values should be used to prognosticate patients for IVF stimulation regimens.

\section{ACKNOWLEDGEMENT}

Chan JKY received salary support from the National Medical Research Council (NMRC/CSA-SI/008-2016), Ministry of Health, Singapore.

\section{REFERENCES}

1. Humaidan P, Kol S, Papanikolaou EG, Copenhagen GnRH Agonist Triggering Workshop Group. GnRH agonist for triggering of final oocyte maturation: time for a change of practice? Hum Reprod Update 2011; 17:510-24.

2. Ling LP, Phoon JW, Lau MS, et al. GnRH agonist trigger and ovarian 
hyperstimulation syndrome: relook at 'freeze-all strategy'. Reprod Biomed Online 2014; 29:392-4.

3. La Marca A, Sunkara SK. Individualization of controlled ovarian stimulation in IVF using ovarian reserve markers: from theory to practice. Hum Reprod Update 2014; 20:124-40.

4. Himabindu Y, Sriharibabu M, Gopinathan K, et al. Anti-mullerian hormone and antral follicle count as predictors of ovarian response in assisted reproduction. J Hum Reprod Sci 2013; 6:27-31.

5. Broer SL, Mol BW, Hendriks D, Broekmans FJ. The role of antimullerian hormone in prediction of outcome after IVF: comparison with the antral follicle count. Fertil Steril 2009; 91:705-14.

6. Broer SL, Dólleman $\mathrm{M}$, Opmeer BC, et al. AMH and AFC as predictors of excessive response in controlled ovarian hyperstimulation: a meta-analysis. Hum Reprod Update 2011; 17:46-54.

7. Dewailly D, Andersen CY, Balen A, et al. The physiology and clinical utility of anti-Mullerian hormone in women. Hum Reprod Update 2014; 20:370-85.

8. Nardo LG, Christodoulou D, Gould D, et al. Anti-Müllerian hormone levels and antral follicle count in women enrolled in in vitro fertilization cycles: relationship to lifestyle factors, chronological age and reproductive history. Gynecol Endocrinol 2007; 23:486-93.

9. van Disseldorp J, Lambalk CB, Kwee J, et al. Comparison of inter- and intra-cycle variability of anti-Mullerian hormone and antral follicle counts. Hum Reprod 2010; 25:221-7.

10. Lee $\mathrm{MH}$, Tar AW, Siew $\mathrm{CH}$, et al. Basal serum anti-müllerian hormone and antral follicle count are predictors of ovarian response for Asian women in Singapore. Asian Pac J Reprod 2014; 3:169-75.

11. Loy SL, Cheung YB, Fortier MV, et al. Age-related nomograms for antral follicle count and anti-Mullerian hormone for subfertile Chinese women in Singapore. PLoS One 2017; 12:e189830.

12. Seifer DB, Golub ET, Lambert-Messerlian G, et al. Variations in serum müllerian inhibiting substance between white, black, and Hispanic women. Fertil Steril 2009; 92:1674-8.

13. Schuh-Huerta SM, Johnson NA, Rosen MP, et al. Genetic variants and environmental factors associated with hormonal markers of ovarian reserve in Caucasian and African American women. Hum Reprod 2012; 27:594-608.

14. La Marca A, Sighinolfi G, Argento C, et al. Polymorphisms in gonadotropin and gonadotropin receptor genes as markers of ovarian reserve and response in in vitro fertilization. Fertil Steril 2013; 99:970-8.e1.

15. Han X, McShane M, Sahertian R, White C, Ledger W. Pre-mixing serum samples with assay buffer is a prerequisite for reproducible anti-Mullerian hormone measurement using the Beckman Coulter Gen II assay. Hum Reprod 2014; 29:1042-8.

16. Nelson SM, La Marca A. The journey from the old to the new AMH assay: how to avoid getting lost in the values. Reprod Biomed Online 2011; 23:411-20.

17. Iliodromiti S, Salje B, Dewailly D, et al. Non-equivalence of anti-Müllerian hormone automated assays: clinical implications for use as a companion diagnostic for individualised gonadotrophin dosing. Hum Reprod 2017;
32:1710-5.

18. Indran IR, Huang Z, Khin LW, et al. Simplified 4-item criteria for polycystic ovary syndrome: a bridge too far? Clin Endocrinol (Oxf) 2018; 89:202-11.

19. Ferraretti AP, La Marca A, Fauser BC, et al; ESHRE working group on Poor Ovarian Response Definition. ESHRE consensus on the definition of 'poor response' to ovarian stimulation for in vitro fertilization: the Bologna criteria. Hum Reprod 2011; 26:1616-24.

20. Broer SL, van Disseldorp J, Broeze KA, et al. Added value of ovarian reserve testing on patient characteristics in the prediction of ovarian response and ongoing pregnancy: an individual patient data approach. Hum Reprod Update 2013; 19:26-36.

21. Nelson SM, Yates RW, Fleming R. Serum anti-Müllerian hormone and FSH: prediction of live birth and extremes of response in stimulated cycles-implications for individualization of therapy. Hum Reprod 2007; 22:2414-21.

22. Reichman DE, Goldschlag D, Rosenwaks Z. Value of antimüllerian hormone as a prognostic indicator of in vitro fertilization outcome. Fertil Steril 2014; 101:1012-8.e1

23. Lan VT, Linh NK, Tuong HM, Wong PC, Howles CM. Anti-Müllerian hormone versus antral follicle count for defining the starting dose of FSH. Reprod Biomed Online 2013; 27:390-9.

24. Eldar-Geva T, Ben-Chetrit A, Spitz IM, et al. Dynamic assays of inhibin B, anti-Mullerian hormone and estradiol following FSH stimulation and ovarian ultrasonography as predictors of IVF outcome. Hum Reprod 2005; 20:31 78-83.

25. van Rooij IA, Broekmans FJ, te Velde ER, et al. Serum anti-Müllerian hormone levels: a novel measure of ovarian reserve. Hum Reprod 2002; 17:3065-71.

26. Ebner T, Sommergruber M, Moser M, et al. Basal level of anti-Müllerian hormone is associated with oocyte quality in stimulated cycles. Hum Reprod 2006; 21:2022-6.

27. Broer SL, Dólleman M, van Disseldorp J, et al; IPD-EXPORT Study Group. Prediction of an excessive response in in vitro fertilization from patient characteristics and ovarian reserve tests and comparison in subgroups: an individual patient data meta-analysis. Fertil Steril 2013; 100:420-9.e7.

28. Lee TH, Liu CH, Huang CC, et al. Serum anti-Müllerian hormone and estradiol levels as predictors of ovarian hyperstimulation syndrome in assisted reproduction technology cycles. Hum Reprod 2008; 23:160-7.

29. Fréour T, Mirallié S, Bach-Ngohou K, et al. Measurement of serum anti-Müllerian hormone by Beckman Coulter ELISA and DSL ELISA: comparison and relevance in assisted reproduction technology (ART). Clin Chim Acta 2007; 375:162-4.

30. Li HW, Ng EH, Wong BP, et al. Correlation between three assay systems for antiMüllerian hormone (AMH) determination. J Assist Reprod Genet 2012; 29:1443-6.

31. Sunkara SK, Rittenberg V, Raine-Fenning N, et al. Association between the number of eggs and live birth in IVF treatment: an analysis of 400,135 treatment cycles. Hum Reprod 2011; 26:1768-74.

32. Steward RG, Lan L, Shah AA, et al. Oocyte number as a predictor for ovarian hyperstimulation syndrome and live birth: an analysis of 256,381 in vitro fertilization cycles. Fertil Steril 2014; 101:967-73. 